Discrete Comput Geom 38:389-397 (2007)

DOI: $10.1007 / \mathrm{s} 00454-007-1343-6$

\title{
The Empty Hexagon Theorem
}

\author{
Carlos M. Nicolás \\ Department of Mathematics, University of Kentucky, \\ Lexington, KY 40506, USA \\ cnicolas@ms.uky.edu
}

\begin{abstract}
Let $\mathrm{P}$ be a finite set of points in general position in the plane. Let $\mathcal{C}(\mathrm{P})$ be the convex hull of $\mathrm{P}$ and let $C_{\mathrm{P}}^{i}$ be the $i$ th convex layer of $\mathrm{P}$. A minimal convex set $\mathrm{S}$ of $\mathrm{P}$ is a convex subset of $\mathrm{P}$ such that every convex set of $\mathrm{P} \cap \mathcal{C}(\mathrm{S})$ different from $\mathrm{S}$ has cardinality strictly less than $|\mathrm{S}|$. Our main theorem states that $\mathrm{P}$ contains an empty convex hexagon if $C_{\mathrm{P}}^{1}$ is minimal and $C_{\mathrm{P}}^{4}$ is not empty. Combined with the Erdôs-Szekeres theorem, this result implies that every set $\mathrm{P}$ with sufficiently many points contains an empty convex hexagon, giving an affirmative answer to a question posed by Erdős in 1977.
\end{abstract}

\section{Introduction}

In 1935 (see [3]) Erdős and Szekeres proved the following important result:

The Erdôs-Szekeres Theorem. For every $k \geq 3$ there exists a minimum positive integer $N(k)$ such that every set of at least $N(k)$ points in general position in the plane (i.e., no three on a line) contains $k$ points which are the vertices of a convex $k$-gon.

In the same paper they made the following conjecture:

The Erdös-Szekeres Conjecture. $\quad N(k)=2^{k-2}+1$.

The best known bounds on $N(k)$ are $2^{k-2}+1 \leq N(k)$ and $N(k) \leq\left(\begin{array}{c}2 k-5 \\ k-2\end{array}\right)+1$ (in [4] and [11], respectively). See [1] and [8] for a survey of problems and results connected to the Erdős-Szekeres theorem.

In 1977 (see [2]) Erdős posed the following related problem:

The Empty Convex Polygon Problem. Is there a minimum positive integer $H(k)$ such that every set $X$ of at least $H(k)$ points in general position in the plane contains 
$k$ points which are the vertices of an empty convex $k$-gon, i.e., the vertices of a convex $k$-gon containing no points of $X$ in its interior?

It is known that $H(4)=5$ and $H(5)=10$ [6]. On the other hand, in 1983 Horton showed that $H(k)$ does not exist for $k \geq 7$ [7]. In [9] Overmars exhibited a set of 29 points, the largest known, with no empty convex hexagons. He also conjectured that any such set can have at most seven extreme points in its convex hull. This conjecture, which turned out to be wrong, was the starting point of our work.

In the present paper we settle the question of the existence of $H(6)$, which is the only case that remained open from the original empty polygon problem. We show that for any finite set of points in general position, if the set contains a sufficiently large convex subset, then it also contains an empty convex hexagon. Therefore, by the Erdós-Szekeres theorem, the result follows:

The Empty Hexagon Theorem. H(6) is finite.

We obtain the bound $H(6) \leq N(25)$. An independent result of Gerken (see [5]) gives the better bound $H(6) \leq N(9)$.

\section{Preliminaries}

Throughout this work, all the sets of points are in general position.

Let $\mathcal{C}(\mathrm{P})$ denote the convex hull of $\mathrm{P}$ and let $E(\mathrm{P})$ be the set of vertices (extreme points) of $\mathcal{C}(\mathrm{P})$. Inductively define the $i$ th convex layer of $\mathrm{P}$ by $C_{\mathrm{P}}^{i}=E\left(\mathrm{P}-\bigcup_{j=1}^{i-1} C_{\mathrm{P}}^{j}\right)$ for $i \geq 1$. A set $\mathrm{P}$ is convex if $\mathrm{P}=C_{\mathrm{P}}^{1}$. An empty convex set of $\mathrm{P}$ is a subset $\mathrm{S}$ of $\mathrm{P}$ such that $\mathrm{S}$ is convex and $\mathcal{C}(\mathrm{S}) \cap \mathrm{P}=\mathrm{S}$. We say that $\mathrm{P}$ contains an empty convex $k$-gon if it contains an empty convex $k$-set.

A convex subset $\mathrm{S}$ of $\mathrm{P}$ is a minimal convex set of $\mathrm{P}$ if every convex subset of $\mathrm{P} \cap \mathcal{C}(\mathrm{S})$ different from $\mathrm{S}$ has cardinality strictly less than $|\mathrm{S}|$.

Given two distinct points $p, q$ of $\mathrm{P}$, let $\mathcal{H}(p, q)$ be the closed half-plane to the left of the directed line through $p$ and $q$. For a sequence $p_{1}, \ldots, p_{k}$ of points in $\mathrm{P}$ with $p_{i} \neq p_{i+1}$, let $\mathcal{H}\left(p_{1}, \ldots, p_{k}\right)=\bigcup_{i=1}^{k-1} \mathcal{H}\left(p_{i}, p_{i+1}\right)$. If $\mathrm{S}$ is a convex set, an edge of $\mathrm{S}$ is any 2-sequence $p, q$ with $p \neq q$ such that $q$ immediately follows $p$ when the elements of $\mathrm{S}$ are listed in cyclic clockwise order. If $p, q$ is an edge of $\mathrm{S}$, we also say that $p$ and $q$ are consecutive.

We begin by showing a simple property that $C_{\mathrm{P}}^{2}$ satisfies when $C_{\mathrm{P}}^{1}$ is minimal.

Theorem 1. If $C_{\mathrm{P}}^{1}$ is a minimal convex set of $\mathrm{P}$ then $\left|\mathcal{H}(p, q) \cap C_{\mathrm{P}}^{1}\right| \geq 3$ for every edge $p, q$ of $C_{\mathrm{P}}^{2}$.

Proof. Suppose $\left|\mathcal{H}(p, q) \cap C_{\mathrm{P}}^{1}\right| \leq 2$. Then $\{p, q\} \cup\left(C_{\mathrm{P}}^{1}-\mathcal{H}(p, q)\right)$ is a convex set contained in $\mathcal{C}\left(C_{\mathrm{P}}^{1}\right)$ with at least as many points as $C_{\mathrm{P}}^{1}$ and different from $C_{\mathrm{P}}^{1}$, contrary to the minimality of $C_{\mathrm{P}}^{1}$ (see Fig. 1(a)). 


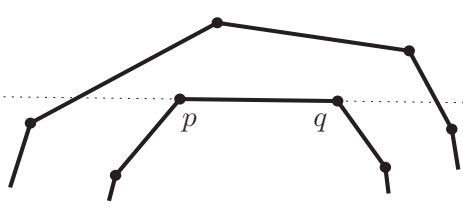

(a)

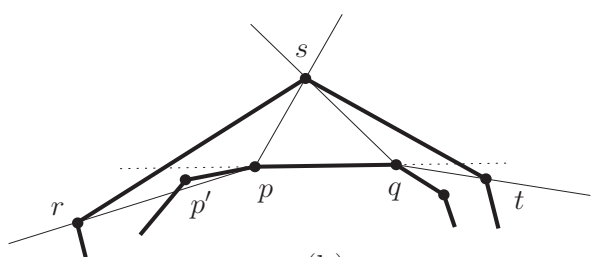

(b)

Fig. 1. Examples for Theorems 1 and 3.

The existence of $H(5)$ follows immediately from Theorem 1.

Theorem 2. $H(5) \leq N(6)$.

Proof. Let $\mathrm{R}$ be a set of points in general position. Suppose $|\mathrm{R}| \geq N(6)$, so that $\mathrm{R}$ contains a convex 6-set. Let $\mathrm{S}$ be a minimal convex 6-set of $\mathrm{R}$. Let $\mathrm{P}=\mathrm{R} \cap \mathcal{C}(\mathrm{S})$. Clearly, $C_{\mathrm{P}}^{1}=\mathrm{S}$ and $C_{\mathrm{P}}^{1}$ is a minimal convex 6-set of $\mathrm{P}$. There are three cases. If $\left|C_{\mathrm{P}}^{2}\right| \geq 2$, let $p$ and $q$ be consecutive points of $C_{\mathrm{P}}^{2}$. By Theorem $1,\left|\mathcal{H}(p, q) \cap C_{\mathrm{P}}^{1}\right| \geq 3$, hence $\{p, q\} \cup\left(\mathcal{H}(p, q) \cap C_{\mathrm{P}}^{1}\right)$ contains an empty convex 5-set of $\mathrm{P}$ and also of $\mathrm{R}$. If $\left|C_{\mathrm{P}}^{2}\right|=1$, let $p \in C_{\mathrm{P}}^{2}$ and $q \in C_{\mathrm{P}}^{1}$. Either $\left|\mathcal{H}(p, q) \cap C_{\mathrm{P}}^{1}\right| \geq 3$ or $\left|\mathcal{H}(q, p) \cap C_{\mathrm{P}}^{1}\right| \geq 3$, therefore $p, q$ and three points of $C_{\mathrm{P}}^{1}$ make an empty convex 5-gon of R. If $\left|C_{\mathrm{P}}^{2}\right|=0$, then $\mathrm{P}$ is an empty convex 6-set of $\mathrm{R}$.

Given distinct points $p, q, r$ of $\mathrm{P}$, let $\mathcal{V}_{r}^{p, q}=\mathcal{H}(p, r) \cap \mathcal{H}(r, q)$.

Theorem 3. If $C_{\mathrm{P}}^{1}$ is a minimal convex set of $\mathrm{P}$ then either $\left|\mathcal{H}(p, q) \cap C_{\mathrm{P}}^{2}\right| \geq 2$ for every edge $p, q$ of $C_{\mathrm{P}}^{3}$, or $\mathrm{P}$ contains an empty convex hexagon.

Proof. Suppose $\left|\mathcal{H}(p, q) \cap C_{\mathrm{P}}^{2}\right| \leq 1$ for some edge $p, q$ of $C_{\mathrm{P}}^{3}$. Clearly, $\mathcal{H}(p, q) \cap C_{\mathrm{P}}^{2}=$ $\{s\}$ for some $s \in C_{\mathrm{P}}^{2}$. Let $r$ and $t$ be the points such that $r, s$ and $s, t$ are edges of $C_{\mathrm{P}}^{2}$ (see Fig. 1(b)). Note $\{r, p, q, t\}$ is convex. If $\left|\mathcal{H}(r, p, q, t) \cap C_{\mathrm{P}}^{1}\right| \leq 4$ then $\{r, p, q, t\} \cup\left(C_{\mathrm{P}}^{1}-\right.$ $\mathcal{H}(r, p, q, t))$ contains a convex $\left|C_{\mathrm{P}}^{1}\right|$-set, contradicting the minimality of $C_{\mathrm{P}}^{1}$. Therefore, $\left|\mathcal{H}(r, p, q, t) \cap C_{\mathrm{P}}^{1}\right| \geq 5$. However, $C_{\mathrm{P}}^{1} \cap \mathcal{H}(r, p, q, t)=C_{\mathrm{P}}^{1} \cap\left(\mathcal{V}_{p}^{r, s} \cup \mathcal{V}_{q}^{s, t}\right)$, so either $\left|C_{\mathrm{P}}^{1} \cap \mathcal{V}_{p}^{r, s}\right| \geq 3$ or $\left|C_{\mathrm{P}}^{1} \cap \mathcal{V}_{q}^{s, t}\right| \geq 3$. Hence, three points of $C_{\mathrm{P}}^{1}$ together with either $r, p^{\prime}, s$ or $s, q^{\prime}, t$ form an empty convex hexagon in $\mathrm{P}$, where $p^{\prime}$ and $q^{\prime}$ are respectively points in $C_{\mathrm{P}}^{3} \cap \mathcal{C}(r, p, s)$ and $C_{\mathrm{P}}^{3} \cap \mathcal{C}(s, q, t)$ such that $\left\{r, p^{\prime}, s\right\}$ and $\left\{s, q^{\prime}, t\right\}$ are empty.

\section{The Proof}

Let $r, r^{\prime}$ and $q, q^{\prime}$ be edges of $C_{\mathrm{P}}^{2}$ and $C_{\mathrm{P}}^{3}$ respectively. We say that $r, r^{\prime}$ and $q, q^{\prime}$ can be matched if the interior of $\mathcal{C}\left(r, r^{\prime}, q, q^{\prime}\right)$ is contained in $\mathcal{C}\left(C_{\mathrm{P}}^{2}\right)-\mathcal{C}\left(C_{\mathrm{P}}^{3}\right)$, where $\mathcal{C}\left(r, r^{\prime}, q, q^{\prime}\right)$ means $\mathcal{C}\left(\left\{r, r^{\prime}, q, q^{\prime}\right\}\right)$. Note that if $r, r^{\prime}$ and $q, q^{\prime}$ can be matched, then $\left\{r, r^{\prime}, q, q^{\prime}\right\}$ is an empty convex 4-set. Similarly, the edge $r, r^{\prime}$ (resp. vertex $r$ ) of $C_{\mathrm{P}}^{2}$ can be matched to the vertex $q$ (the edge $q, q^{\prime}$ ) of $C_{\mathrm{P}}^{3}$ if the interior of $\mathcal{C}\left(r, r^{\prime}, q\right)$ (of $\left.\mathcal{C}\left(r, q, q^{\prime}\right)\right)$ is contained in $\mathcal{C}\left(C_{\mathrm{P}}^{2}\right)-\mathcal{C}\left(C_{\mathrm{P}}^{3}\right)$. 
Now let $r, r^{\prime}$ and $r^{\prime}, r^{\prime \prime}$ be two consecutive edges of $C_{\mathrm{P}}^{2}$ that can be matched to the edges $q, q^{\prime}$ and $q^{\prime \prime}, q^{\prime \prime \prime}$ of $C_{\mathrm{P}}^{3}$, respectively. These matchings are order-preserving if the interiors of $\mathcal{C}\left(r, r^{\prime}, q, q^{\prime}\right)$ and $\mathcal{C}\left(r^{\prime}, r^{\prime \prime}, q^{\prime \prime}, q^{\prime \prime \prime}\right)$ are disjoint. Note that in this case we obtain that the regions $\mathcal{H}(r, q) \cap \mathcal{H}\left(q, q^{\prime}\right) \cap \mathcal{H}\left(q^{\prime}, r^{\prime}\right)$ and $\mathcal{H}\left(r^{\prime}, q^{\prime \prime}\right) \cap \mathcal{H}\left(q^{\prime \prime}, q^{\prime \prime \prime}\right) \cap \mathcal{H}\left(q^{\prime \prime \prime}, r^{\prime \prime}\right)$ overlap in the complement of $C_{\mathrm{P}}^{2}$. Also note that a similar statement holds if we match any (or both) of the edges in $C_{\mathrm{P}}^{2}$ to a vertex in $C_{\mathrm{P}}^{3}$. Therefore, if we match every edge in $C_{\mathrm{P}}^{2}$ to an edge or vertex in $C_{\mathrm{P}}^{3}$ in such a way that all the matchings of consecutive edges of $C_{\mathrm{P}}^{2}$ are order-preserving, then the union of the induced regions completely covers the complement of $C_{\mathrm{P}}^{2}$, and consequently it contains $C_{\mathrm{P}}^{1}$. In what follows, every matching of consecutive edges of $C_{\mathrm{P}}^{2}$ is going to be order-preserving, a fact that is easy to check and that justifies all the claims about $C_{\mathrm{P}}^{1}$ being covered by regions induced by given matchings.

The next theorem is our main result.

Theorem 4. If $C_{\mathrm{P}}^{1}$ is a minimal convex set of $\mathrm{P}$ and $C_{\mathrm{P}}^{4} \neq \emptyset$ then $\mathrm{P}$ contains an empty convex hexagon.

Proof. Let $p \in C_{\mathrm{P}}^{4}$. Let $C_{\mathrm{P}}^{3}=\left\{q_{0}, \ldots, q_{m-1}\right\}$ and $C_{\mathrm{P}}^{2}=\left\{r_{0}, \ldots, r_{n-1}\right\}$ with $q_{i}, q_{i+1}$ and $r_{j}, r_{j+1}$ consecutive for all $i, j$. The indices $i$ and $j$ in $q_{i}$ and $r_{j}$ should always be read modulo $m$ and $n$, respectively. Inequalities of the form $k \leq i \leq k^{\prime}$, involving indices of the points $\left\{q_{i}\right\}$, signify $i \in\left\{k, \ldots, k^{\prime}\right\}$ in the case $k \leq k^{\prime}$, and $i \in\{\mathrm{k}, \ldots, \mathrm{m}$ $1\} \cup\left\{0, \ldots, k^{\prime}\right\}$ in the case $k^{\prime}<k$. Inequalities involving the points $\left\{r_{j}\right\}$ are defined in a similar way.

Clearly $C_{\mathrm{P}}^{2} \subset \bigcup_{i} \mathcal{V}_{p}^{q_{i}, q_{i+1}}$. For each $j \in\{0, \ldots, n-1\}$, let $i_{j}$ be the unique index in $\{0, \ldots, m-1\}$ such that $r_{j} \in \mathcal{V}_{p}^{q_{i_{j}}, q_{i_{j}+1}}$.

We say that $r_{j}$ can be matched to the left if $r_{j-1}, r_{j}$ and $q_{i_{j}}, q_{i_{j}+1}$ can be matched. Similarly, $r_{j}$ can be matched to the right if $r_{j}, r_{j+1}$ can be matched to $q_{i_{j}}, q_{i_{j}+1}$. Let $\mathcal{L}^{r_{j}}=\mathcal{H}\left(r_{j-1}, q_{i_{j}}\right) \cap \mathcal{H}\left(q_{i_{j}}, q_{i_{j}+1}\right) \cap \mathcal{H}\left(q_{i_{j}+1}, r_{j}\right)$. Let $\mathcal{R}^{r_{j}}=\mathcal{H}\left(r_{j}, q_{i_{j}}\right) \cap \mathcal{H}\left(q_{i_{j}}, q_{i_{j}+1}\right) \cap$ $\mathcal{H}\left(q_{i_{j}+1}, r_{j+1}\right)$. When $r_{j}$ can be matched to the left, the set $\mathcal{L}^{r_{j}}$ satisfies the following property that will be used repeatedly: if $\left|C_{\mathrm{P}}^{1} \cap \mathcal{L}^{r_{j}}\right| \geq 2$ then $\mathrm{P}$ contains an empty convex hexagon. The set $\mathcal{R}^{r_{j}}$ satisfies a similar property, provided that $r_{j}$ can be matched to the right. Also note that if $r_{j_{0}}$ cannot be matched neither to the left nor to the right for some $j_{0}$, then there exists an empty convex hexagon by Theorem 3 .

Now we single out a situation that constitutes the core of this proof.

Case I. Suppose there exist $b, k \in\{0, \ldots, n-1\}$ such that:

(i) $i_{b}<i_{b+1}<\cdots<i_{b+k}$.

(ii) $r_{b+h}$ can be matched to the right for every $0 \leq h \leq k-1$.

(iii) $r_{b}$ cannot be matched to the left.

(iv) $r_{b+k}$ cannot be matched to the right.

Without loss of generality, assume in addition that $b=0$.

Note that under the present hypothesis $C_{\mathrm{P}}^{2} \cap \mathcal{V}_{p}^{q_{i}, q_{i_{j}+1}}=\left\{r_{j}\right\}$ for every $j$ in $\{0, \ldots, k\}$. In particular, $\left|\left\{i_{j}, 0 \leq j \leq k\right\}\right|=k+1$. In order to show that these conditions imply the existence of an empty hexagon, we need to distinguish three cases depending on $k$ : 


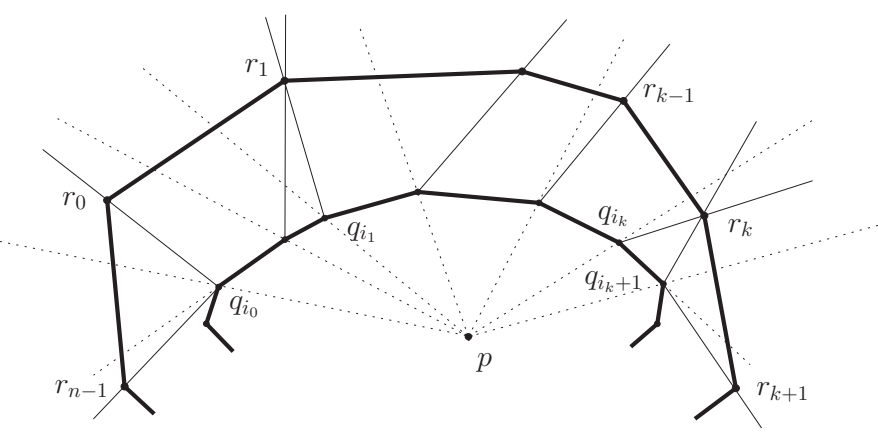

Fig. 2. Case I.A.

Case I.A: $0 \leq k \leq n-3$. Note that for $k=0$ we get $\mathcal{H}\left(q_{i_{0}}, q_{i_{0}+1}\right) \cap C_{\mathrm{P}}^{2}=\left\{r_{0}\right\}$, so $\mathrm{P}$ contains an empty hexagon by Theorem 3 .

The bounds on $k$ together with (i), (iii) and (iv) imply that $\mathrm{T}=\left\{r_{n-1}, r_{k+1}\right\} \cup\left\{q_{i}, i_{0} \leq\right.$ $\left.i \leq i_{k}+1\right\}$ is a convex set with at least $k+4$ elements. Note that $r_{n-1}$ and $r_{k+1}$ are distinct since $k \leq n-3$.

Let $\mathrm{L}$ be the sequence $r_{n-1}, q_{i_{0}}, q_{i_{1}}, \ldots, q_{i_{k}}, q_{i_{k}+1}, r_{k+1}$. See Fig. 2 .

If $\left|\mathcal{H}(\mathrm{L}) \cap C_{\mathrm{P}}^{1}\right| \leq k+4$, then $\mathrm{T} \cup\left(C_{\mathrm{P}}^{1}-\mathcal{H}(\mathrm{L})\right)$ contains a convex $\left|C_{\mathrm{P}}^{1}\right|$-set, contradicting the minimality of $C_{\mathrm{P}}^{1}$. Therefore, $\left|\mathcal{H}(\mathrm{L}) \cap C_{\mathrm{P}}^{1}\right| \geq k+5$.

Now,

$$
\mathcal{H}(\mathrm{L}) \cap C_{\mathrm{P}}^{1} \subset \mathcal{V}_{q_{i_{0}}}^{r_{n-1}, r_{0}} \cup \bigcup_{j=0}^{k-1} \mathcal{R}^{r_{j}} \cup \mathcal{V}_{q_{i_{k}+1}}^{r_{k}, r_{k+1}} .
$$

For each $j$ satisfying $\left|C_{\mathrm{P}}^{1} \cap \mathcal{R}^{r_{j}}\right| \geq 2$, we obtain an empty convex hexagon. If $\mid C_{\mathrm{P}}^{1} \cap$ $\mathcal{R}^{r_{j}} \mid \leq 1$ for $0 \leq j \leq k-1$, then $\left|C_{\mathrm{P}}^{1} \cap\left(\mathcal{V}_{q_{i 0}}^{r_{n-1}, r_{0}} \cup \mathcal{V}_{q_{i_{k}+1}}^{r_{k}, r_{k+1}}\right)\right| \geq 5$. We are not done since $r_{n-1}, r_{0}, q_{i_{0}}$ or $r_{k}, r_{k+1}, q_{i_{k}+1}$ might not be empty; for example, it could happen that $q_{i_{0}-1} \in \mathcal{C}\left(r_{n-1}, r_{0}, q_{i_{0}}\right)$. Choosing $q^{\prime}$ and $q^{\prime \prime}$ satisfying that $r_{n-1}, r_{0}, q^{\prime}$ and $r_{k}, r_{k+1}, q^{\prime \prime}$ are empty and $q^{\prime} \in C_{\mathrm{P}}^{3} \cap \mathcal{C}\left(r_{n-1}, r_{0}, q_{i_{0}}\right), q^{\prime \prime} \in C_{\mathrm{P}}^{3} \cap \mathcal{C}\left(r_{k}, r_{k+1}, q_{i_{k}+1}\right)$, we get that three points of $C_{\mathrm{P}}^{1}$ and $r_{n-1}, r_{0}, q^{\prime}$ or $r_{k}, r_{k+1}, q^{\prime \prime}$ make an empty convex hexagon.

Case I.B: $k=n-2$. Let $h=i_{k+1}$, so $r_{k+1} \in \mathcal{H}\left(q_{h}, q_{h+1}\right)$. See Fig. 3 .

By Theorem 3, we assume that $\left|C_{\mathrm{P}}^{2} \cap \mathcal{H}\left(q_{h}, q_{h+1}\right)\right| \geq 2$. Therefore, either $r_{k+1}, r_{0}$ or $r_{k}, r_{k+1}$ can be matched to $q_{h}, q_{h+1}$. Both cases can be handled in the same way, so suppose that $r_{k+1}$ can be matched to the left, that is, $r_{k}, r_{k+1}$ and $q_{h}, q_{h+1}$ can be matched.

From (i), (iii) and (iv) it follows that $\left\{r_{k+1}, q_{i_{0}}, q_{i_{1}}, \ldots, q_{i_{k}}, q_{i_{k}+1}\right\}$ is a convex ( $k+3$ )set contained in $C_{\mathrm{P}}^{1}$, so necessarily $\left|C_{\mathrm{P}}^{1}\right| \geq k+4$, by the minimality of $C_{\mathrm{P}}^{1}$. Now, using (ii),

$$
C_{\mathrm{P}}^{1} \subset \mathcal{V}_{q_{i_{0}}}^{r_{k+1}, r_{0}} \cup \bigcup_{j=0}^{k-1} \mathcal{R}^{r_{j}} \cup \mathcal{L}^{r_{k+1}} .
$$

If $\left|C_{\mathrm{P}}^{1} \cap \mathcal{R}^{r_{j}}\right| \geq 2$ (for some $0 \leq j \leq k-1$ ) or $\left|C_{\mathrm{P}}^{1} \cap \mathcal{L}^{r_{k+1}}\right| \geq 2$, we are done. If not, then $\left|C_{\mathrm{P}}^{1} \cap \mathcal{V}_{q_{0}}^{r_{k+1}, r_{0}}\right| \geq 3$, so three points of $C_{\mathrm{P}}^{1}$ and $r_{k+1}, r_{0}, q^{\prime}$ form an empty convex hexagon for some $q^{\prime} \in C_{\mathrm{P}}^{3} \cap \mathcal{C}\left(r_{k+1}, r_{0}, q_{i_{0}}\right)$. 


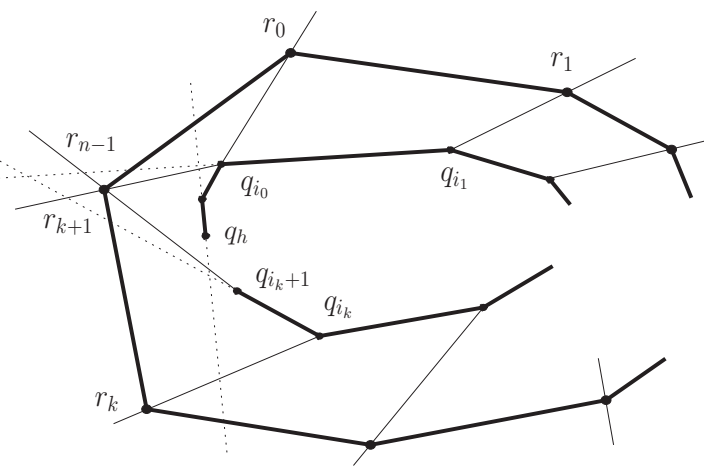

Fig. 3. Case I.B.

Case I.C: $k=n-1$. Since $C_{\mathrm{P}}^{1} \subset \mathcal{V}_{q_{i}}^{r_{k}, r_{0}} \cup \bigcup_{j=0}^{k-1} \mathcal{R}^{r_{j}}$, we get an empty hexagon if $\left|C_{\mathrm{P}}^{1}\right| \geq k+3$. Henceforth, we assume that $\left|C_{\mathrm{P}}^{1}\right| \leq k+2$.

Note that if $i_{j}<i_{j}+1<i_{j+1}$ for some $0 \leq j \leq k-1$, then $\left|C_{\mathrm{P}}^{3}\right| \geq k+2$, contradicting the minimality of $C_{\mathrm{P}}^{1}$. Therefore, $i_{j+1}=i_{j}+1$ for $0 \leq j \leq k-1$. Also, by the same reason, $i_{k}+1=i_{0}$. Consequently $\left|C_{\mathrm{P}}^{3}\right|=k+1$ and $\left|C_{\mathrm{P}}^{1}\right|=k+2$.

Certainly $r_{k} \in \mathcal{H}\left(q_{i_{k}}, q_{i_{0}}\right)$ since $r_{k} \in \mathcal{V}_{p}^{q_{i_{k}}, q_{i_{0}}}$. By Theorem 3 there is another point of $C_{\mathrm{P}}^{2}$ in $\mathcal{H}\left(q_{i_{k}}, q_{i_{0}}\right)$. However, $r_{k}, r_{0}$ and $q_{i_{k}}, q_{i_{0}}$ cannot be matched (by (iv)), so necessarily $r_{0} \notin \mathcal{H}\left(q_{i_{k}}, q_{i_{0}}\right)$. Therefore, $r_{k-1}$ belongs to $\mathcal{H}\left(q_{i_{k}}, q_{i_{0}}\right)$ which implies that $r_{k}$ can be matched to the left. See Fig. 4.

Consider the line through $q_{i_{0}-1}$ and $q_{i_{0}+1}$. If both $r_{1}$ and $r_{k-1}$ lie to the right of this line, that is, if $\left\{r_{1}, r_{k-1}\right\} \subset \mathcal{H}\left(q_{i_{0}+1}, q_{i_{0}-1}\right)$, then $\left\{r_{k-1}, q_{i_{0}-1}, q_{i_{0}+1}, r_{1}\right\}$ is convex. Using the minimality of $C_{\mathrm{P}}^{1}$ as before we get that $\left|C_{\mathrm{P}}^{1} \cap \mathcal{H}(\mathrm{L})\right| \geq 5$, where $\mathrm{L}$ is the sequence $r_{k-1}, q_{i_{0}-1}, q_{i_{0}+1}, r_{1}$. However,

$$
C_{\mathrm{P}}^{1} \cap \mathcal{H}(\mathrm{L}) \subset \mathcal{L}^{r_{k}} \cup \mathcal{V}_{q_{i_{0}}}^{r_{k}, r_{0}} \cup \mathcal{R}^{r_{0}},
$$

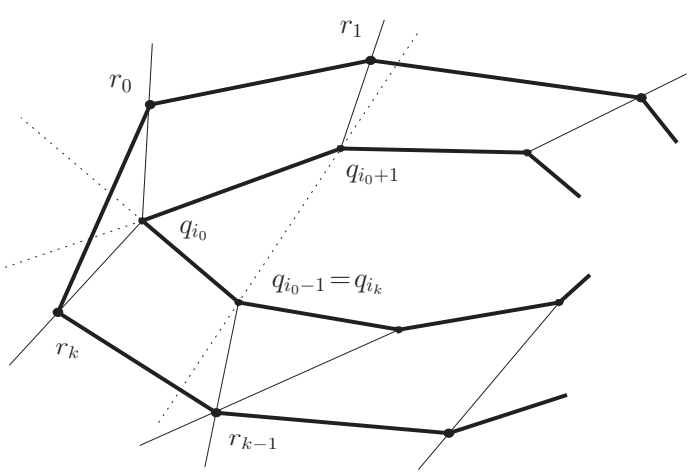

Fig. 4. Case I.C. 
therefore $\left|C_{\mathrm{P}}^{1} \cap \mathcal{L}^{r_{k}}\right| \geq 2$ or $\left|C_{\mathrm{P}}^{1} \cap \mathcal{V}_{q_{i_{0}}}^{r_{k}, r_{0}}\right| \geq 3$ or $\left|C_{\mathrm{P}}^{1} \cap \mathcal{R}^{r_{0}}\right| \geq 2$. In every case we obtain an empty convex hexagon.

Now suppose that $\left\{r_{1}, r_{k-1}\right\} \not \subset \mathcal{H}\left(q_{i_{0}+1}, q_{i_{0}-1}\right)$. Without loss of generality, assume that $r_{1} \notin \mathcal{H}\left(q_{i_{0}+1}, q_{i_{0}-1}\right)$.

These conditions imply that $\mathcal{R}^{r_{0}} \subset \mathcal{V}_{q_{i_{k}}}^{q_{i_{0}}, q_{i_{0}+1}}$, which in turn implies that $\mathrm{P}$ contains an empty convex hexagon when $\left|C_{\mathrm{P}}^{1} \cap \mathcal{R}^{r_{0}}\right| \geq 1$, using a point $p^{\prime} \in C_{\mathrm{P}}^{4} \cap \mathcal{C}\left(q_{i_{0}}, q_{i_{0}+1}, q_{i_{k}}\right)$ in place of $q_{i_{k}}$ if $\left\{q_{i_{0}}, q_{i_{0}+1}, q_{i_{k}}\right\}$ is not empty. Now,

$$
C_{\mathrm{P}}^{1} \subset \mathcal{V}_{q_{i_{0}}}^{r_{k}, r_{0}} \cup \mathcal{R}^{r_{0}} \cup \bigcup_{j=1}^{k-1} \mathcal{R}^{r_{j}} .
$$

However, $\left|C_{\mathrm{P}}^{1}\right|=k+2$, so $\left|C_{\mathrm{P}}^{1} \cap \mathcal{V}_{q_{i}}^{r_{k}, r_{0}}\right| \geq 3$ or $\left|C_{\mathrm{P}}^{1} \cap \mathcal{R}^{r_{0}}\right| \geq 1$ or $\left|C_{\mathrm{P}}^{1} \cap \mathcal{R}^{r_{j}}\right| \geq 2$ for some $1 \leq j \leq k-1$. Again in each case we get an empty convex hexagon.

Case II. Assume Case I does not hold.

This situation can be broken down into three cases according to the maximum number of points of $C_{\mathrm{P}}^{2}$ in the sets $\mathcal{V}_{p}^{q_{i}, q_{i+1}}$.

Case II.A: If $\max _{i}\left|C_{\mathrm{P}}^{2} \cap \mathcal{V}_{p}^{q_{i}, q_{i+1}}\right|=1$. If $r_{j}$ can be matched to the left for every $j$, then

$$
C_{\mathrm{P}}^{1} \subset \bigcup_{j=0}^{n-1} \mathcal{L}^{r_{j}}
$$

By the minimality of $C_{\mathrm{P}}^{1}$ we have that $\left|C_{\mathrm{P}}^{1}\right| \geq n+1$ since $\left|C_{\mathrm{P}}^{2}\right|=n$. Hence $\left|C_{\mathrm{P}}^{1} \cap \mathcal{L}^{r_{h}}\right| \geq 2$ for some $h$ by the pigeonhole principle. However, this implies that two points of $C_{\mathrm{P}}^{1}$ together with $r_{h-1}, r_{h}, q_{i_{h}}$ and $q_{i_{h}+1}$ form an empty convex hexagon. The situation is exactly the same if $r_{j}$ can be matched to the right for every $j$.

If none of the above happens, then $r_{b}$ cannot be matched to the left for some $0 \leq b \leq$ $n-1$. Take $k \geq 0$ to be the maximum integer such that $r_{b+h}$ can be matched to the right for every $0 \leq h \leq k-1$. Then necessarily $0 \leq k \leq n-1$ and $r_{b+k}$ cannot be matched to the right. Therefore we are back in Case I, contrary to our assumption.

Case II.B: If $\max _{i}\left|C_{\mathrm{P}}^{2} \cap \mathcal{V}_{p}^{q_{i}, q_{i+1}}\right|=2$.

Let $D=\left\{j \in\{0, \ldots, n-1\} \mid C_{\mathrm{P}}^{2} \cap \mathcal{V}_{p}^{q_{i j}, q_{i_{j}+1}}=\left\{r_{j-1}, r_{j}\right\}\right\}$.

Let $\bar{j} \in D$, so $i_{\bar{J}-1}=i_{\bar{j}}$. Clearly, $r_{\bar{J}}$ can be matched to the left and $i_{\bar{J}} \neq i_{\bar{J}+1}$.

Let $t=\max \left\{0 \leq t^{\prime} \leq n-1 \mid i_{\bar{J}+h} \neq i_{\bar{j}+h+1}\right.$ for all $\left.0 \leq h \leq t^{\prime}\right\}$.

Let $s=\max \left\{0 \leq s^{\prime} \leq t \mid r_{j+h}\right.$ can be matched to the left for all $\left.0 \leq h \leq s^{\prime}\right\}$.

If $s<t$, then $r_{\bar{J}+s+1}$ cannot be matched to the left. By Theorem 3, we may assume it can be matched to the right. Let $g=\max \left\{s+1 \leq g^{\prime} \leq t \mid r_{\bar{J}+h}\right.$ can be matched to the right for all $s+1 \leq h \leq g^{\prime}$ \}.

We claim that necessarily $g=t$. Suppose $g<t$. Then, taking $b=\bar{J}+s+1$ and $k=g-s$, the four conditions of Case I are satisfied, contrary to our assumption.

Hence $r_{j+h}$ can be matched to the left for all $h \in\{0, \ldots, s\}$, and to the right for all $h \in\{s+1, \ldots, t\}$. Note that only the edge $r_{\bar{J}+s}, r_{\bar{j}+s+1}$ remains to be matched, which 
can be done using a vertex in $C_{\mathrm{P}}^{3}$. Let $\bar{l}, i_{\bar{J}+s}+1 \leq \bar{\imath} \leq i_{\bar{J}+s+1}$, be such that $r_{\bar{J}+s}, r_{\bar{j}+s+1}$ and $q_{\bar{l}}$ can be matched.

Therefore,

$$
C_{\mathrm{P}}^{1} \subset \bigcup_{\bar{j} \in D}\left(\mathcal{L}^{r_{j}} \cup \bigcup_{h=1}^{s} \mathcal{L}^{r_{j+h}} \cup \mathcal{V}_{q_{\bar{i}}}^{r_{j+s}, r_{j+s+1}} \cup \bigcup_{h=s+1}^{t} \mathcal{R}^{r_{j+h}}\right) .
$$

In this formula, as in the forthcoming paragraphs, the quantities $s, t$ and $\bar{l}$ are dependent on $\bar{J}$, though we write $s, t, \bar{l}$ instead of $s(\bar{J}), t(\bar{J}), \bar{l}(\bar{J})$ for the sake of simplicity.

If $\mathcal{L}^{r_{j}}$ contains a point of $C_{\mathrm{P}}^{1}$ then that point together with $r_{\bar{J}-1}, r_{\bar{j}}, q_{i_{j}}, q_{i_{j}+1}$ and $p^{\prime}$ form an empty convex hexagon for some $p^{\prime} \in \mathrm{P} \cap \mathcal{C}\left(q_{i_{\bar{j}}}, q_{i_{j}+1}, p\right)$. Therefore we may assume that $\left|C_{\mathrm{P}}^{1} \cap \mathcal{L}^{r_{j}}\right|=0$ for all $\bar{j} \in D$. For similar reasons, we assume that $\left|C_{\mathrm{P}}^{1} \cap \mathcal{L}^{r_{j+h}}\right| \leq 1$ for all $1 \leq h \leq s$, and $\left|C_{\mathrm{P}}^{1} \cap \mathcal{R}^{r_{j+h}}\right| \leq 1$ for all $s+1 \leq h \leq t$, so the sets $\mathcal{L}^{r_{j+h}}$ and $\mathcal{R}^{r_{j+h}}$ contain at most $\sum_{j \in D} t$ points of $C_{\mathrm{P}}^{1}$.

However, $\left|C_{\mathrm{P}}^{2}\right|=2|D|+\sum_{j \in D} t$ and $C_{\mathrm{P}}^{1}$ contains at least one more point because it is minimal.

Therefore $\left|C_{\mathrm{P}}^{1} \cap \bigcup_{\bar{j} \in D} \mathcal{V}_{q_{\bar{i}}}^{r_{j+s}, r_{j+s+1}}\right| \geq 1+2|D|$, so $\left|C_{\mathrm{P}}^{1} \cap \mathcal{V}_{q_{\bar{i}}}^{r_{j^{\prime}+s}, r_{j^{\prime}+s+1}}\right| \geq 3$ for some $\bar{J}^{\prime} \in D$, which implies that $\mathrm{P}$ contains an empty convex hexagon.

Case II.C: If $\max _{i}\left|C_{\mathrm{P}}^{2} \cap \mathcal{V}_{p}^{q_{i}, q_{i+1}}\right| \geq 3$. Take $k$ such that $\left|C_{\mathrm{P}}^{2} \cap \mathcal{V}_{p}^{q_{k}, q_{k+1}}\right| \geq 3$. Hence, three points of $C_{\mathrm{P}}^{2}$ together with $q_{k}, q_{k+1}$ and $p^{\prime}$ form an empty convex hexagon, where $p^{\prime}$ is any point in $\mathrm{P} \cap \mathcal{C}\left(q_{k}, q_{k+1}, p\right)$ such that $\left\{q_{k}, q_{k+1}, p^{\prime}\right\}$ is an empty 3-set.

The finiteness of $H(6)$ follows immediately from Theorem 4.

Corollary. $H(6) \leq N(25)$.

Proof. Let R be a finite set of points in general position. Suppose $|\mathrm{R}| \geq N(25)$, so that $\mathrm{R}$ contains a convex 25 -set. Let $\mathrm{S}$ be a minimal convex 25 -set of $\mathrm{R}$ and let $\mathrm{P}=\mathrm{R} \cap \mathcal{C}(\mathrm{S})$. Clearly, $C_{\mathrm{P}}^{1}=\mathrm{S}$ and $C_{\mathrm{P}}^{1}$ is a minimal convex 25 -set of P. Let $C_{\mathrm{P}}^{2}=\left\{r_{0}, \ldots, r_{n-1}\right\}$ and $C_{\mathrm{P}}^{3}=\left\{q_{0}, \ldots, q_{m-1}\right\}$ with $r_{j}, r_{j+1}$ and $q_{i}, q_{i+1}$ consecutive for all $i, j$.

By Theorem 4, P contains an empty convex hexagon if $C_{\mathrm{P}}^{4} \neq \emptyset$.

If $C_{\mathrm{P}}^{4}=\emptyset$ then $C_{\mathrm{P}}^{3}$ is an empty $m$-gon, so we may assume that $m \leq 5$. If $C_{\mathrm{P}}^{3}=\emptyset$ then $C_{\mathrm{P}}^{2}$ is empty, and if we assume $n \leq 5$ we get an empty heptagon since $C_{\mathrm{P}}^{1} \subset$ $\bigcup_{j=0}^{n-1} \mathcal{H}\left(r_{j}, r_{j+1}\right)$.

If $C_{\mathrm{P}}^{3} \neq \emptyset$ then $C_{\mathrm{P}}^{1} \subset \bigcup_{j=0}^{n-1} \mathcal{V}_{q_{0}}^{r_{j}, r_{j+1}}$, so necessarily $\left|C_{\mathrm{P}}^{1} \cap \mathcal{V}_{q_{0}}^{r_{j^{\prime}}, r_{j^{\prime}+1}}\right| \geq 3$ for some $j^{\prime}$ when $n \leq 12$, since $\left|C_{\mathrm{P}}^{1}\right|=25$. Consequently there exists an empty convex hexagon with a vertex in $C_{\mathrm{P}}^{3} \cap \mathcal{C}\left(q_{0}, r_{j^{\prime}}, r_{j^{\prime}+1}\right)$. If $n \geq 13$ we find an empty hexagon between $C_{\mathrm{P}}^{2}$ and $C_{\mathrm{P}}^{3}$ because $C_{\mathrm{P}}^{2} \subset \bigcup_{i=1}^{m-2} \mathcal{V}_{q_{0}}^{q_{i}, q_{i+1}} \cup \mathcal{H}\left(q_{m-1}, q_{0}\right) \cup \mathcal{H}\left(q_{0}, q_{1}\right)$, therefore we have that $\left|C_{\mathrm{P}}^{1} \cap \mathcal{V}_{q_{0}}^{q_{i}, q_{i+1}}\right| \geq 3$ or $\left|C_{\mathrm{P}}^{1} \cap \mathcal{H}\left(q_{m-1}, q_{0}\right)\right| \geq 4$ or $\left|C_{\mathrm{P}}^{1} \cap \mathcal{H}\left(q_{0}, q_{1}\right)\right| \geq 4$, and in every case we get an empty hexagon.

Any upper bound on $H(6)$ gives a linear lower bound for the number of empty convex hexagons in the set R. More precisely, let $g_{k}(\mathrm{R})$ be the number of empty convex $k$-sets contained in R. Let $f_{k}(n)=\min \left\{g_{k}(\mathrm{R})|| \mathrm{R} \mid=n\right\}$. Note that $f_{7}(n)=0$ by Horton's result. It is not difficult to find quadratic lower bounds for $f_{3}(n)$ and $f_{4}(n)$, while it is 
still an open problem to determine if there is a superlinear lower bound for $f_{5}(n)$, see [1] and [10]. Now we can also ask: Is there a superlinear lower bound for $f_{6}(n)$ ?

\section{References}

1. P. Brass, W. Moser and J. Pach, Research Problems in Geometry, Springer, New York, 2005.

2. P. Erdős, Some applications of graph theory and combinatorial methods to number theory and geometry, Algebraic Methods in Graph Theory, Vols. I, II (Szeged, 1978), pp. 137-148, North-Holland, Amsterdam, 1981.

3. P. Erdős and G. Szekeres, A combinatorial problem in geometry, Compos. Math. 2 (1935), 463-470. Reprinted in: Paul Erdôs: The Art of Counting. Selected Writings (J. Spencer, ed.), pp. 3-12, MIT Press, Cambridge, MA, 1973

4. P. Erdős and G. Szekeres, On some extremum problems in elementary geometry, Ann. Univ. Sci. Budapest. Eötvös Sect. Math. 3-4 (1961), 53-62. Reprinted in: Paul Erdös: The Art of Counting. Selected Writings (J. Spencer, ed.), pp. 680-689, MIT Press, Cambridge, MA, 1973.

5. T. Gerken, Empty convex hexagons in planar point sets, submitted to Discrete Comput. Geom.

6. H. Harboth, Konvexe Fünfecke in ebenen Punktmengen, Elem. Math. 33 (1978), 116-118.

7. J.D. Horton, Sets with no empty convex 7-gon, Canad. Math. Bull. 26 (1983), 482-484.

8. W. Morris and V. Soltan, The Erdős-Szekeres problem on points in convex position, a survey, Bull. Amer. Math. Soc. 37 (2000), 437-458.

9. M.H. Overmars, Finding sets of points without empty convex 6-gons, Discrete Comput. Geom. 29 (2003), 153-158.

10. R. Pinchasi, R. Radoičić and M. Sharir, On empty convex polygons in a planar point set, J. Combin. Theory Ser. A 113 (2006), 385-419.

11. G. Tóth and P. Valtr, The Erdős-Szekeres theorem, upper bounds and generalizations, Discrete and Computational Geometry —Papers from the MSRI Special Program (J. E. Goodman et al., eds.), Cambridge University Press, Cambridge, 2005.

Received September 28, 2005, and in revised form March 13, 2006. Online publication July 13, 2007. 